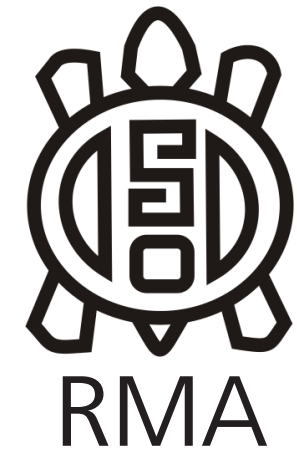

Antropología Social

\title{
Un mundo de músicas instrumentales. Aproximaciones sobre escenas de jazz-rock de la ciudad de Córdoba entre 1978/1997
}

\author{
A word of instrumental music. Approaches to jazz-rock scenes in the \\ city of Córdoba between 1978/1997 \\ María Sol Bruno*
}

*Centro de Investigaciones María Saleme de Burnichón, Facultad de Filosofía y Humanidades, Universidad Nacional de Córdoba. Argentina.

E-mail: mariasolbruno@yahoo.com.ar

\begin{abstract}
Resumen
En este artículo indagamos una escena musical de la ciudad de Córdoba donde las producciones eran mayormente instrumentales, y pertenecieron a un género musical que fusionaba el rock y el jazz. Nos preguntamos por las formas y características que tomaron aquellas poéticas a partir de la trayectoria de dos conjuntos musicales integrados por artistas en común: el grupo Encuentro (1978/1982) y Los Músicos del Centro (1982/1997). Este texto, como señala el título, es una aproximación empírica, que carece de antecedentes locales que remitan específicamente al género del jazz o a los conjuntos abordados. El trabajo se estructura a través de tres ejes centrales. Para empezar, realizamos una breve presentación de los artistas, elementos distintivos de sus trayectorias, consumos culturales y formación musical. Posteriormente analizamos las producciones sonoras que se materializaron en discos de los dos grupos musicales. Por último, ahondamos sobre los sistemas de distribución y formas de financiamiento de las obras de arte.
\end{abstract}

Palabras clave: Mundos de arte; Músicas; Jazz-Rock; Córdoba; Argentina.

\begin{abstract}
In this article we investigate a music scene in Córdoba city, where the productions were mostly instrumental, and belonged to a musical genre that fused rock and jazz. We wonder about the forms and characteristics that those poetics took from de two musical groups made up of artists in common: Grupo Encuentro (1978/1982) and Los Músicos del Centro (1982/1997). This text is an empirical approach, without local antecedents that refer specifically to the jazz genre or the groups addressed. The work is structured through three central axes. In the beginning, we make a brief presentations of the artists, distinctive elements of their careers, cultural consumption and musical training. Later we analyze the sound productions that were materialized in discs of the two musical groups. Finally, we delve into de distribution systems and forms of financing works of art.
\end{abstract}

Key words: Arts Word; Music; Jazz-Rock; Cordoba; Argentina.

\section{Introducción}

En la tesis de doctorado investigamos producciones musicales en la ciudad de Córdoba (Bruno, 2019). Analizamos una compleja trama de relaciones interdependientes (Elias, 1987) que se configuraron en redes de cooperación que posibilitaron la producción de músicas entre 1979 y 1992. A partir de las herramientas conceptuales de Howard Becker (2008) delimitamos un mundo de arte, una red colaborativa, diversa en estilos y géneros, que permitió la circulación y producción de creaciones musicales. Dentro de esta trama diferenciamos escenas (Bennet \& Peterson, 2004; Straw, 1972) a partir de las cuales agrupamos las producciones sonoras. Una escena es un ensamblaje que permite a productores, músicos y públicos compartir producciones musicales, son espacios donde coexisten un rango de prácticas musicales que interactúan mediante una variedad de procesos de diferenciación. En este texto ponemos la mirada en una escena de músicas instrumentales. ${ }^{1}$

\footnotetext{
1 Somos conscientes que tomamos autores de tradiciones teóricas diferentes, esta convergencia responde tanto a mi formación en historia, estudios culturales y en antropología, como al acompañamiento de un equipo de investigación que incentivó estas lecturas. Combinar estos autores permitió en la tesis de doctorado poner en consideración diferentes aspectos analíticos. A modo de síntesis podríamos decir que Elias nos recordó que es pertinente no perder de vista los contextos históricos en la producción artística, Becker incentivó abrir la mirada a los procesos de producción artística, Bennet, Peterson y Straw ofreció una posible delimitación de la complejidad de la vasta producción artística de la época que abrumaba a la propia etnógrafa por su diversidad y variabilidad. Aunque no es objetivo de este artículo
} 
Esta escena se diferenciaba de otras abordadas en la investigación porque privilegiaban las músicas por sobre las canciones. Las producciones eran mayormente instrumentales, las poesías y letras aparecían esporádicamente de la mano de artistas invitados como compositores e intérpretes. En este artículo nos preguntamos por las formas que tomaron aquellas poéticas a partir de la trayectoria de dos conjuntos musicales integrados por artistas en común. Nos referimos al grupo Encuentro (1978/1982) y a LoS Músicos del Centro (1982/1997).

Estos artistas se abocaban a un género musical que fusionaba el rock y el jazz. En los inicios de la formación de estos conjuntos, el rock era un género musical en expansión en el país destinado principalmente a sectores juveniles, que alcanzaría su clímax de difusión comercial luego de la guerra de Malvinas en 1982. El jazz tenía un público selecto y menor en la ciudad de Córdoba. Esta situación, se replicaba en la ciudad de Buenos Aires, aunque promediando la década de 1970 el género se expandió hacia otras exploraciones sonoras más allá de los clásicos standards (Pujol, 1992). Nuevos compositores se lanzaron a la tarea creativa, que combinó sus saberes con otros espacios de la música como el rock, el tango y el folklore. Fue en este contexto que emergen los conjuntos que exploramos en este texto. Asimismo, cabe destacar que desde la perspectiva de los interlocutores los músicos de jazz contaban con un preciado tesoro que no circulaba en otros medios. Los músicos de jazz tenían mayor (in) formación musical: conocían sobre armonía, ritmos, trucos de improvisación, enlaces, repertorios y formas de interpretación que los distinguía de otros conjuntos contemporáneos.

Este artículo, como señala su propio título, es una aproximación que carece de antecedentes locales que remitan específicamente al género del jazz. Cabe señalar que se cuenta con trabajos que abordan otros mundos musicales de la ciudad como la música del cuarteto (Blázquez, 2008, 2014), música electrónica (Blázquez, 2019), música interpretada por DJ mujeres (Rodríguez, 2018), música folklórica (Acurso, 2016), hip hop (Vittoreli, 2019) o trabajos de autoría propia. Asimismo, existen antecedentes a nivel nacional que remiten al estudio de la música de jazz (Corti, 2015, 2018; Karush, 2017; López, 2016; Pujol, 1992, 2013, 2015, 2020) y a otros géneros (Alabarces, 1993; Benzecry, 2012; Buch, 2016; Díaz, 2005,2009; Lenarduzzi, 2012; Manzano, 2011; Molinero \& Vila, 2014; Pujol, 2005, 2011; Semán, 2012, Semán \& Vila, 1999).

El artículo se estructura a través de tres ejes centrales. Para empezar, realizamos una breve presentación de los artistas, elementos distintivos de sus trayectorias, consumos culturales y formación musical. Posteriormente analizamos

particular, considero que podrían profundizarse estas discusiones en otros espacios académicos las producciones sonoras que se materializaron en discos de los dos conjuntos. Por último, ahondamos sobre los sistemas de distribución y formas de financiamiento de las obras de arte. Como planteamos, este artículo recupera fragmentos de la tesis doctoral, aunque para este texto en particular cabe aclarar que trabajamos con entrevistas en profundidad con miembros de esta escena de jazz-rock y con las obras registradas en discos que gentilmente fueron cedidas por los entrevistados. ${ }^{2}$

\section{Sobre los artistas: trayectorias, aprendizajes, gustos musicales}

Una pista iniciática para indagar la escena de músicos instrumentales fue que, como etnógrafa, entrevisté a docentes universitarios que estudiaron en la Universidad Nacional de Córdoba. A partir de entrevistas, describí una serie de itinerarios y consumos culturales que efectuaban un conjunto de personas durante su periodo estudiantil. Se trataba de un grupo de amistad que se conformó durante la década de 1970, cuando los jóvenes cursaban en una misma institución sus estudios secundarios. Estas relaciones se sostuvieron en el tiempo, cuando muchos de ellos comenzaron sus estudios superiores. Una actividad que celebraba ese vínculo de amor fue devenir público, y personal de apoyo de un conjunto instrumental que formó Domingo "Mingui" Ingaramo. Así es que un grupo de jóvenes se encontraban asiduamente en las veredas de sus domicilios a escuchar discos de sus propios amigos y a organizar su presencia en locales nocturnos mercantilizados de la ciudad de Córdoba. ${ }^{3}$

Ingaramo (n. 1958) es oriundo de la ciudad de Córdoba, y la eligió como su lugar de residencia hasta el momento que realizamos la tesis. Se inició en la práctica musical en su niñez, cuando comenzó a estudiar piano con un profesor particular. Este instrumento se encontraba disponible en la casa donde vivía, su padre era músico y

\footnotetext{
2 Cabe aclarar que para lo que se pretende abordar en este artículo no analizamos fuentes documentales más allá de los discos. Deseamos continuar y profundizar la pesquisa en próximos proyectos, para lo cual la revisión de la prensa especializada y no especializada de la época será una de las primeras actividades a realizar. Nos centramos en entrevistas en profundidad porque fue pertinente para el análisis de este artículo en particular. Todas las entrevistas referidas fueron realizadas por la autora, se trató de entrevistas no estructuradas con una serie de preguntas establecidas de antemano pero que se fueron modificando de acuerdo al entrevistado. Las personas fueron contactadas mediante la técnica de bola de nieve, lo cual habilitaba un vínculo de confianza con los interlocutores. El trabajo de campo se inició en el año 2010 con el objetivo de concretar mi tesina de grado, por lo cual los vínculos establecidos con las personas entrevistadas en muchas ocasiones trascendieron el espacio de la entrevista en particular y se continúan en el presente mediante invitaciones, correos electrónicos, redes sociales o llamadas telefónicas. Esta cercanía habilita la construcción de saberes, pero también propone otros desafíos, algunas dificultades pueden saldarse con la triangulación de datos con fuentes documentales y otras requieren de reflexiones metodológicas más profundas.

3 Resulta preciso aclarar que en este artículo no se abordan los públicos y consumos de las obras musicales en cuestión por razones de extensión. Esta variable será retomada en futuros escritos para profundizar el análisis.
} 
pianista de una orquesta típica. Tanto "Mingui" como su hermano mayor Juan Carlos (n. 1953) se transformaron en ejecutantes de este instrumento, como una especie de herencia de una tradición familiar. Su práctica y estudio fue acompañada con un profesor particular que les enseñaba música académica.

Además del piano, Ingaramo señaló, en la entrevista que sostuvimos con él, que en su niñez tuvo la posibilidad de acceder a diferentes músicas que repercutieron en su posterior profesión como artista. Los discos y la guía auditiva provinieron de su padre, quien le hizo conocer obras de tango, pero principalmente de su hermano mayor Juan Carlos con quien integraría luego los proyectos musicales a los cuales referimos en este artículo. La música elegida resultaba de un complejo proceso donde intervenían otras personas, en general de mayor edad y/o que ocupaban espacios con mayor información.

A partir de este proceso de selección Ingaramo se inclinó por la audición de conjuntos de rock internacional como The Beatles, Pink Floyd o Emerson, Like \& Palmer; jazz como Jaco Pastorius, Chick Corea, Herbie Hancock; música brasilera como João Gilberto, Milton Nascimento o Ivan Lins, o de Uruguay como los hermanos Fattoruso. Para este interlocutor todas estas músicas eran "músicas populares con altísimo nivel de formación estético, música buena, copada y no comercial" (Entrevista con M. Ingaramo, 10 de abril de 2014, Córdoba). Esta forma de describir los consumos y gustos musicales, respondía a complejos procesos de distinción. Estos juicios estéticos tenían repercusiones en la formación de grupos sociales, pues se vinculaban con relaciones de amistad y noviazgo. Pero también funcionaban como indicadores sobre los espacios que los sujetos imaginaban que ocupaban dentro de los mundos musicales (Frith, 2014). Aunque en este artículo no pretendemos indagar en los contrastes entre diferentes escenas musicales de la ciudad de Córdoba, en el trabajo de campo que realizamos para la tesis doctoral, hallamos que entre los interlocutores había un universo musical que ellos despreciaban y se constituía como una alteridad que marcaba fronteras morales. Esta diferenciación por contraste puede ayudar a dar cuenta de la especificidad de una escena instrumental en la ciudad de Córdoba, y responder el interrogante: ¿cuáles eran las características de estas producciones musicales que las diferenciaban de otras contemporáneas?

Cuando eran adolescentes los hermanos Ingaramo hicieron sus primeros ensayos como grupo musical. Como ambos eran pianistas, "Mingui" se inició como guitarrista. Para aprender este nuevo instrumento no tomó clases particulares. Escuchaba música, sacaba temas de oído y hacía ejercicios de técnica. El aprendizaje autodidacta sin la presencia de docentes que guiaran la enseñanza puede haberse vinculado con el saber previo de otro instrumento por parte del artista. Sin embargo, no descartamos su predisposición y estudio individual que posibilitó la performance como guitarrista.

No obstante, la condición de autodidacta no significó que el aprendizaje musical fuera una acción exclusivamente individual. Como las producciones artísticas no son independientes de la existencia social de sus creadores (Elias, 1999), los artistas se integran en diferentes grupos y configuran encuentros entre otros miembros de la sociedad que ocupan diferentes espacios en los mundos del arte. Para Ingaramo, resultaba importante compartir y tocar música con otros jóvenes músicos. Estos espacios de encuentro eran propicios para intercambiar información sobre artistas admirados, nuevos artistas, discos y teoría musical. Los datos circulaban por redes de conocidos, y cuando tomaban contacto con ellos eran calificados como un "tesoro". En palabras del entrevistado:

\begin{abstract}
"Entonces era como... viste Paul McCarney cuenta que con Lennon cuando eran chiquitos, se tomaron un ómnibus y atravesaron todo Londres a buscar un pibe que le habian dicho que tocaba el C7ma, una posición de acordes que eran las séptimas. ${ }^{4} Y$ dice, porque fuimos $y$ llegamos a la casa y el tipo tocaba, no [cara de asombro]. Y no y volvimos. Esos tesoros ¿viste? Ese tipo de cosas. Y en aquella época era así cuando escuchaba un guitarrista o un disco era lo que llegaba de afuera... Con el jazz se fue armando la constelación de información que uno iba necesitando para enamorarse cada vez más de lo que hacía y al mismo tiempo no era como ahora que tenés acceso a todo, de todo." (Entrevista con M. Ingaramo)
\end{abstract}

Una técnica que utilizaba Ingaramo para contactarse con otros músicos era llamar a la puerta en caso que, al caminar por las veredas de su barrio, escuchara la práctica del algún instrumento. En ocasiones los alumnos de los músicos oficiaban de mediadores y colaboraban para contactar a las personas. Tanto Ingaramo, como otros artistas, al cumplir con cierta trayectoria y desempeño, se iniciaban en la docencia. El dictado de clases particulares en sus hogares les permitía un ingreso económico extra para llevar adelante sus proyectos artísticos. Al mismo tiempo conocían otros jóvenes interesados en la música que oficiaban de intermediarios y públicos de sus producciones.

Dentro de estas redes de sociabilidad aparecieron, en el relato de la trayectoria de Ingaramo, músicos que se convirtieron en maestros de los cuales aprender el

\footnotetext{
4 Esta notación hace referencia a la triada mayor construida a partir de la nota do, con el agregado del intervalo de la séptima menor. El uso de las séptimas constituye un aprendizaje por parte de los músicos que requiere entrenamiento y estudio, y forma parte de los conocimientos que se agrupan en lo que se entiende como armonía en música. El uso de séptimas resultan fundamentales para ejecutar lo que se llama función dominante-tónica, una secuencia de dos acordes que tienen un fuerte efecto conclusivo en la música occidental.
} 
oficio musical. Estas personas tenían la particularidad de contar con mayor información musical, formación y perfeccionamiento de ejecución. Eran músicos que podían desenvolverse como instrumentistas con gran maestría. En palabras de Ingaramo, se trataba de "músicos avezados y experimentados".

En la formación de estos artistas, la circulación de información entre pares y sobre todo pares "mayores" era fundamental. Al respecto advertimos una percepción "evolucionista" de los interlocutores, pues el mayor tiempo acumulado en la vida de las personas era significado como portadores de saberes y conocimientos ausentes en sujetos de edades biológicas menores. Las instituciones de enseñanza de música o las clases con profesores particulares no volvieron a tener importancia en la trayectoria de Ingaramo. Su aprendizaje se continuó por su cuenta, un estudio en la privacidad de su hogar que se complementaba con la performance colectiva con artistas de mayor formación y experiencia.

Detectamos que, en sintonía con los músicos de jazz que analizan Becker \& Faulkner (2011), estos artistas aprendían los temas que deseaban ejecutar a partir de oírlos en grabaciones, de discos o la radio, o representaciones en vivo que presenciaban, ya sea conciertos públicos o encuentros privados en casas particulares. A partir de un entrenamiento y una conducta repetida de escucha atenta, los músicos lograban tocar la melodía y acertar a los patrones armónicos de los temas que deseaban. Como señalan los autores, esta situación que vuelve "automática" su práctica y les permite improvisaciones sin tener errores técnicos les lleva mucho tiempo de entrenamiento de oído y repetición previa. El aprendizaje de oído también les posibilitaba apreciar detalles de ejecución particulares, más allá de la melodía y armonía, como fraseos, rítmicas y estilos de interpretación.

Un artista importante en la trayectoria de los Ingaramo fue Daniel Homer (n. 1948). Homer vivía en el mismo barrio, y provenía de una familia de músicos. Comenzó a estudiar guitarra en el Conservatorio Provincial de la ciudad, donde se destacó como alumno. Si bien no concluyó sus estudios allí, continuó su formación con profesores particulares. Durante la década de 1960 integró diferentes bandas de pop/rock/beat. En el año 1967, gracias a la intermediación de su hermano mayor, también músico, emprendió una gira como parte de la banda de César Isella por diferentes países latinoamericanos. ${ }^{5}$ En la entrevista que le realizamos (28 de julio de 2014, Ciudad Autónoma

5 Isella era un cantautor de música folklórica y uno de los integrantes del movimiento del Nuevo Cancionero. El movimiento de la Nueva Canción nació en Mendoza en 1963 con la publicación de un manifiesto que proponía la renovación de la música folclórica. Aglutinó a una serie de artistas (músicos y poetas) enmarcados en una corriente de pensamiento crítica a la tradición liberal y que se conoció como "izquierda nacional". Bregaban por un posicionamiento "(...) antiimperialista que debía buscar sus raíces en la cultura latinoamericana" (Lucero, 2009, p 91). Sus artistas más representativos fueron Armando Tejada Gómez y Hamlet Lima Quintana. de Buenos Aires), Homer recordó su contacto con los hermanos Ingaramo a principios de la década de 1970, antes de mudarse a Buenos Aires en el año 1972.

Ya instalado en la ciudad de Buenos Aires, Homer se desempeñó como productor artístico de la compañía EMI Odeón, durante nueve años. De manera simultánea integró bandas de reconocidos artistas internacionales y fue músico sesionista. ${ }^{6}$ A pesar de la distancia geográfica, Homer siguió en contacto con Córdoba, tanto por motivos afectivos como laborales, incluso participó en la grabación de varios discos de artistas cordobeses. Este caso muestra como artistas de Córdoba tejían redes entre sus coterráneos que remontaba el vínculo a relaciones anteriores. Que Homer viviera en la capital del país, trabajara en una compañía internacional y construyera una carrera artística con músicos internacionales posibilitaba que músicos que producían desde Córdoba generaran otros vínculos laborales. Este artista era un intermediario que facilitaba contactos y conexiones que trascendían los estilos y géneros musicales.

Otro músico importante mencionado en la trayectoria artística de los hermanos Ingaramo fue Julián "Pelusa" Navarro (n. 1949). Navarro estudió piano con el mismo maestro, aunque ellos no se encontraron gracias a esta coincidencia. Julián tenía unos años más que los hermanos Ingaramo, cuando se conocieron él había completado su carrera de ingeniero aeronáutico y trabajaba como tal en el Instituto de Investigaciones Aeronáuticas y Espaciales. Paralelamente se desempeñaba en la producción musical asociado a sellos discográficos. Fue este último espacio el que propició el encuentro.

Junto a Navarro los hermanos Ingaramo conformaron el grupo Encuentro en 1978. ${ }^{7}$ Mientras transcurrían los ensayos, ambos hermanos estudiaban arquitectura en la Universidad Nacional de Córdoba. Mingui abandonó luego de dos años, mientras que Juan Carlos se recibió e hizo de aquella profesión una fuente de ingresos para los años que siguieron.

Tanto Homer como Navarro trabajaron como productores en compañías internacionales. Ellos hacían confluir vínculos entre miembros de un mundo de arte, pues las obras artísticas requieren de redes de relaciones que se alimentan de recomendaciones y reconocimiento entre sus integrantes (Becker, 2008). En este sentido resultaba fundamental tener mayor edad/experiencia/conocimiento

\footnotetext{
6 Entre otros, trabajó con Rubén Rada, Raúl Porchetto, Donna Caroll, Banana Pueyrredón, Roque Narvaja, G.I.T, Pimpinela. También realizó grabaciones en Nueva York con músicos de renombre que integraron conjuntos como Weather Report o que tocaban en la banda de Bill Evans.

7 Completaban la formación de este conjunto "Pichón" Bustos, "Lito" Loyola y "Bebe" Caniza. Esta conformación se modificó años después cuando grabaron su segundo trabajo discográfico, Loyola y Bustos dejaron el grupo, en su reemplazo se incorporaron César Franov y Hugo Ordanini. Datos reconstruidos de los discos del conjunto y notas de difusión en el diario La Voz del Interior.
} 
pero también haber construido redes de reconocimiento.

Con el Grupo Encuentro estos músicos se fueron de gira a EEUU. Tuvieron la posibilidad de trabajar con artistas de reconocimiento internacional y alta difusión comercial como Paz Martinez o Pimpinela, lo cual les permitió el vínculo con otros artistas argentinos como Pedro Aznar, Alejandro Lerner, Litto Nebbia, Fito Paéz o Luis Alberto Spinetta. Entre estos músicos, los hermanos Ingaramo, construyeron una relación laboral y afectiva. Así, cuando músicos reconocidos del emergente rock nacional viajaban a Córdoba, presenciaban performance en vivo de estos músicos instrumentales y compartían reuniones privadas en casas particulares. Estos intercambios habilitaron relaciones de reconocimiento artístico mutuo y participaciones como artistas invitados en los trabajos discográficos.

Además de estos intercambios entre artistas, los integrantes del grupo Encuentro accedieron a instrumentos musicales de gran calidad que no eran habituales en conjuntos cordobeses. En ocasiones, los jóvenes compraban los instrumentos en el exterior durante sus giras artísticas, otra posibilidad era intercambiar equipos entre artistas de Buenos Aires. Al respecto, Ingaramo recordó una situación particular cuando ellos adquirieron un equipo de Luis Alberto Spinetta a cambio de un automóvil que era propiedad de ambos hermanos: "Mi viejo nos había regalado un fiat 600 cuando éramos chicos y se lo cambiamos por un piano Rhodes eléctrico a Spinetta. Que estaba todo viejo me acuerdo" (Entrevista con M. Ingaramo).

El Grupo Encuentro hacía presentaciones en vivo en locales comerciales nocturnos de la ciudad. Uno de ellos, llamado Rock y Pop, se convirtió en emblemático, pues los músicos se convocaban allí con una frecuencia semanal. Otro conjunto de similares características denominado Mousse tocaba periódicamente en este espacio. Ambos conjuntos dedicaban parte de sus recitales a realizar performances colectivas con gran contenido de improvisación. Estas prácticas generaron la inquietud de conformar una nueva agrupación entre músicos de las dos bandas llamada Los Músicos del Centro. ${ }^{8}$

Los Músicos del Centro fue un conjunto local que se popularizó a nivel nacional, grabaron varios discos hasta el año 1997. Sus integrantes variaron a lo largo de los años, los hermanos Ingaramo fueron los músicos estables que participaron en todos los trabajos discográficos. Integrantes de este conjunto también intervinieron

8 El grupo Mousse fue contemporáneo a Encuentro. Aunque algunos de sus integrantes se unieron a Los Músicos del Centro este conjunto continuó en actividades y tuvo varias formaciones. Grabaron un disco que bautizaron Mousse en el año 1991. Registros sonoros y poéticas de obras pueden encontrarse en la recopilación documental: La pisada del Unicornio. Relevamiento de testimonio, militancia y memoria. CD. Teatro por la identidad, Abuelas de Plaza de Mayo. 2006. En próximas indagaciones profundizaremos sobre este conjunto, tanto con análisis documental, como entrevistas en profundidad con los artistas. como artistas invitados de otros músicos reconocidos a nivel nacional e internacional. A nivel individual se desempeñaron como músicos de sesión en estudios de Buenos Aires. Con el avanzar de los años, estos artistas obtuvieron trabajos estables en la capital del país como profesionales de la música, aunque no instalaron su domicilio en esta ciudad.

\section{Sobre producciones sonoras que llegaron a discos}

Tanto el grupo Encuentro como Los Músicos del Centro plasmaron parte de sus obras artísticas en discos grabados. Esta situación no era habitual en los mundos musicales de la ciudad de Córdoba de la época que no fueran parte del género cuarteto. ${ }^{9}$ Grabar un disco requería de una inversión e interés de sellos disqueros de capitales internacionales con sedes en la ciudad de Buenos Aires.

El Grupo Encuentro concretó dos trabajos discográficos con el sello Polygram, propiedad de la compañía multinacional Philips Record. El primer disco salió a dos años de la formación del conjunto en 1980 y el segundo poco tiempo antes de la disolución en 1982. El primero se denominó Fábulas de Cielo y el segundo Nuestro Hogar. Ambos trabajos discográficos tuvieron un arte de tapa que no privilegiaba la figura de los músicos del conjunto, solo la contratapa del segundo disco mostraba a los músicos en situación de ejecución. Las dos obras que conformaron el arte de tapa fueron de autoría de Gustavo Brandán, artista plástico local que participó en el diseño de otros discos de artistas que investigamos en la tesis doctoral, como en el diseño de afiches de difusión de festivales y recitales de la época. ${ }^{10}$

En cuanto a las obras sonoras, ambos trabajos discográficos se caracterizaron por poseer obras instrumentales con estilos sonoros que se acercaban al rock sinfónico y fusionaban elementos del jazz. Solo grabaron una canción en Fábulas del Cielo con un texto breve de gran contenido metafórico. Destacamos que los integrantes del grupo

\footnotetext{
9 El cuarteto es un género de música bailable asociada a los sectores populares (Blázquez, 2008, 2014). Muchos conjuntos que desarrollaban sus actividades en la ciudad de Córdoba que no formaron parte de este entramado musical no registraron sus obras en discos. Otros llegaron a concretar dichos proyectos aunque es destacable que los conjuntos que abordamos en este artículo lo hicieron con mayor premura y tuvieron una producción más prolífica que sus contemporáneos. Cabe considerar que particularmente me interesa realizar una exploración sobre la industria musical en la ciudad de Córdoba. Como se mencionó, no se cuentan con antecedentes específicos sobre esta temática, una investigación que dé cuenta de una historización de sellos grabadores en la ciudad de Córdoba podría resultar provechosa. Asimismo, permitiría explorar otra de las variables analíticas propuesta por Howard Becker en los mundos del arte. 10 Gustavo Brandán (n. 1952) es oriundo de la ciudad de Córdoba. Estudió en la Escuela de Artes de la UNC y obtuvo el título de Licenciado en Grabado. Sus producciones se vincularon con el expresionismo, la gráfica, instalaciones, objetos y experiencias digitales (Moyano, 2005). Brandán realizó su primera exposición individual en 1976 en Córdoba, mismo año que presentara tu tesis de Licenciatura y un año después que fuera premiado por el Premio Nacional Dr. Genaro Pérez.
} 
compusieron la totalidad de las obras grabadas, aunque es notable que en el segundo trabajo se diversifican las autorías ya que todos los ejecutantes tenían una composición propia. Esta variedad de compositores quizás se explique por el tiempo transcurrido y las horas de trabajo de los artistas, pues a medida que los músicos se desempeñan como profesionales adquieren mayor destreza técnica y compositiva.

Los Músicos del Centro fue un conjunto con mayor trayectoria, que editó cinco trabajos discográficos. Una particularidad que se dio entre ambos conjuntos que analizamos en este texto es que en 1982 podemos registrar un solapamiento de proyectos. Al parecer, aquel año fue un momento de transición, pues en el mismo trabajo discográfico del Grupo Encuentro se señala que quienes participan son integrantes de los Músicos del Centro. Otro dato relevante es que el primer disco de Los Músicos del Centro se editó en 1982, con el mismo sello discográfico. El trabajo se denominó Volumen 1, los artistas que participaron fueron los mismos que en Nuestro Hogar con la incorporación de dos artistas invitados que participarían en años siguientes de proyectos del conjunto. Incluso el arte de tapa del nuevo disco utiliza un montaje de fotografías, que remite a una misma toma correspondiente a la contratapa del último disco del Grupo Encuentro.

A diferencia de los discos de Encuentro, este primer disco de Los Músicos del Centro privilegia el nombre del conjunto en el arte de tapa. Y por primera vez aparecen los artistas a través de un montaje de fotografías de los músicos tomadas durante una presentación en vivo por Lucía Seguí. ${ }^{11}$ La postura corporal captada en el momento los retrata en una especie de transe y disfrute en plena performance. Los músicos volverán a aparecer en tapa nuevamente en el tercer disco, denominado Ecuador. Sin embargo, solo se retrata a los hermanos Ingaramo en un primer plano, donde solo puede verse sus rostros con una mirada hacia abajo. El arte de tapa de este álbum fue un diseño de Miguel "Mick" Camaño. ${ }^{12}$

En lo que respecta a las tapas de los otros tres discos del conjunto, advertimos estéticas realizadas a partir de montajes y fotografías que no tiene a los artistas como protagonistas. El disco Noventa y Nueve es un montaje

11 Lucía Seguí es una fotógrafa reconocida en el mundo de la música local, sobre todo en lo que refiere al género estilístico del rock. Ella realizó varias fotografías que los propios artistas eligieron para las tapas o contratapa de sus discos. Por otro lado, a partir de la exploración de fuentes que llevamos a cabo encontramos que Seguí realizaba exposiciones de sus trabajos en festivales de rock como La Falda y Córdoba Rock

12 Camaño es artista plástico y músico. Vivió su niñez y adolescencia en el barrio de San Vicente y estudió en la Facultad de Arquitectura y diseño de la UNC como los hermanos Ingaramo. Como artista plástico trabajó para los estudios de Disney y Hanna Barbera en EEUU. Como músico se desempeña como guitarrista y líder de la banda de blues local Ilamada Crosstown Traffic, proyecto que inició en la década de 1990 y que continuaba en actividades hasta los días que escribimos este artículo. de imágenes cuyo diseño estuvo a cargo, nuevamente, en manos del artista plástico Gustavo Brandán junto a Ana Estela Rozzi. Como en los diseños anteriores no es posible detectar referencias geográficas claras, sino una señal que remite al espacio urbano, pues la imagen principal es un auto diseñado para circular en ciudades.

La tapa del cuarto disco, tiene como imagen principal una fotografía del artista madrileño Ricardo Murad. ${ }^{13}$ En dicha imagen aparecen cuatro niños en actitud de camaradería, retratadas en un espacio urbano. Resulta llamativo el contraste racial, entre niños notablemente blancos y rubios, y otros de piel oscura y características fenotípicas afro. La elección del arte de tapa, puede vincularse con la condición racializada del género jazzístico (Corti, 2015; Pujol, 1992). Por último, el arte de tapa de Luminosa emula un afiche callejero sobre un muro de una ciudad. Como el primer disco, las letras ocupan toda la superficie.

Como señalamos, el último disco del Grupo Encuentro y el primero de los Músicos del Centro se editaron en el mismo año. Sin embargo, el segundo trabajo de los Músicos del Centro se demoró siete años en salir. Como los discos que siguieron no tuvieron un intervalo tan prolongado, creemos que este dato requiere que indaguemos los motivos. A partir de los objetos materiales (los discos) notamos que a partir del segundo trabajo emerge un cambio significativo. En el disco noventa y nueve, el sello discográfico a cargo de la distribución ya no será uno de los gigantes de la industria musical con financiación internacional. El disco noventa y nueve, como los que saldrán luego, tiene una nueva imagen en sus tapas, con un logo desconocido hasta entonces: una semicorchea completaba una " $\mathrm{M}$ ", que en conjunto completaban el nombre de "Melopea". Melopea fue un sello independiente pionero en el país: tenía una propuesta novedosa desde la perspectiva de los artistas, productores y públicos.

Litto Nebbia fue el principal impulsor de Melopea a finales del año 1988. Dicho emprendimiento se encargaba de la producción y edición de un catálogo variado en artistas y géneros que, según la descripción de presentación oficial de la página de la empresa, conforman "música argentina de calidad". El vínculo de los integrantes de los Músicos del Centro con Nebbia se remonta a años anteriores a la salida de sus primeros discos con Melopea. Nebbia estuvo a cargo de la producción del disco Volumen 1, y participó del primer tema del álbum como autor y cantante. Esta colaboración como artista invitado se continuó en los siguientes dos discos del conjunto.

Las vinculaciones con Nebbia también se plasmaron en obras discográficas de este artista. Los Músicos del Centro participaron en dos discos: Llegamos de los

13 Este artista realizó otros trabajos para la discográfica Melopea, para Lito Vitale y Litto Nebbia, así como también para Path Metheny en 1993. Ver https://www.360cities.net/es/profile/ricardo-murad consultada 31/03/2021. 
Barcos editado en 1982 y En vivo en Obras de 1983. Ambos trabajos salieron a la venta a través del sello internacional RCA, e implicaron giras al interior del país. Para aquellos años Nebbia era un artista reconocido, fundamentalmente en el rock. ${ }^{14}$ Esta construcción de redes de (re)conocimiento permitieron a los músicos locales participar de un proceso de construcción de reputaciones, pues devenir en acompañantes de un artista consagrado les otorgaba prestigio y proyección nacional.

Las vinculaciones y trabajos cooperativos con Nebbia tuvieron una continuidad, no sólo porque Los Músicos del Centro grabaron sus siguientes discos en el sello del artista rosarino. Entre los artistas se erigieron redes afectivas y laborales que permitieron intercambios y colaboraciones en conciertos en vivo o en otros discos. Por mencionar un ejemplo, en el año 1994 los hermanos Ingaramo grabaron un nuevo trabajo junto a Nebbia titulado Nueva York es una ciudad solitaria.

Volviendo a los trabajos discográficos de Los Músicos del Centro, identificamos que en el primer disco las autorías se dividieron entre dos integrantes del conjunto: Julián Navarro y Juan Carlos Ingaramo. Ya en el segundo trabajo, las autorías quedaron en manos exclusivamente de los hermanos Ingaramo quienes se perfilaron como los dos integrantes fijos del conjunto hasta su disolución. A lo largo de las formaciones registradas en los discos es posible distinguir una variación de los integrantes. El disco Ecuador expresa en la ficha técnica que los integrantes del conjunto son los hermanos Ingaramo junto a Gabriel Pérez y Fernando Bobarini. Sin embargo, la estética del disco, tanto en la portada como en el dorso, muestra fotografiados a ambos Ingaramo. La decisión artística de visibilizar sólo a dos de los músicos responde a una jerarquización en el trabajo de los artistas del conjunto. Tanto Mingui como Juan Carlos fueron los autores de los temas, se encargaron de los arreglos y tocaban más instrumentos en cada una de las pistas. A esta formación se sumaban una extensa lista de artistas invitados. Algunos músicos habían sido parte anteriormente de la formación del conjunto y habían participado en sus discos, o se habían desempeñado como invitados o productores. En el último disco esta situación se revierte, pues si bien

\footnotetext{
14 Nebbia era co-autor de La Balsa, canción que se situó en el mito de origen del rock en el país, y por tanto era uno de los pioneros en el campo (Díaz, 2005). A fines de la década de 1970, el músico conformó un trío de jazz que lo llevó a extender las posibilidades armónicas de sus propias composiciones, a la vez que incorporó influencias musicales de la música brasilera, el tango moderno y el folklore de proyección. Paralelamente perfeccionó la composición letrística auxiliado de Mirtha Defilpo, su pareja de aquellos años. En 1978 decidió exiliarse en México, pues había sido incorporado a las "listas negras" confeccionadas por los dictadores de entonces, tuvo inconvenientes en presentaciones de medios de comunicación, recitales que le fueron suspendidos, sus discos dejaron de pasarse por radio, sufrió una detención y retención de documentación. En 1981 regresó al país, el reencuentro con su público se realizó en un recital durante junio en el estadio Obras (Pujol, 2005). Para mediados de los ochenta Nebbia se situaba dentro del conjunto de "viejos consagrados" (Díaz, 2005).
}

los hermanos Ingaramo son autores de la mayoría de las obras, aparecen nuevos autores: músicos del medio local asociados a la escena del jazz cordobés como Luis Lewin y Javier Girotto.

Si bien en la totalidad de las discos hay una primacía de temas instrumentales, en todos los trabajos se incluye al menos una canción cantada. La particularidad, es que ninguno de los integrantes de Los Músico del Centro tomaba el rol de intérprete en el canto, ni de composición de la letra. Para ello recurrían a la figura de los artistas invitados, quienes simultáneamente eran co-autores de las composiciones. Los artistas invitados tenían una trayectoria artística previa dentro del género del rock argentino, se trataba de figuras como Litto Nebbia, Pedro Aznar, Fito Paéz o Lalo de los Santos. Estos músicos habían participado en diferentes conjuntos importantes y estaban construyendo su carrera solista con reconocimiento no sólo nacional sino también internacional. Habían grabado discos, participado como músicos invitados de otros artistas aún más reconocidos que ellos y se presentaban con asiduidad en diferentes conciertos. Vendían discos y eran entrevistados por la prensa y elogiados por la crítica.

La participación de este tipo de artistas reconocidos en un trabajo discográfico de un conjunto de menor popularidad y prestigio otorgaba mayor valor al disco. Esta colaboración respondía a la construcción redes de relaciones que posibilitaba que los artistas conocieran los trabajos y confiaran en el éxito de dicho proyecto. Pero también se insertaba dentro de la creación de reputaciones que se desarrollaban a partir de un proceso de consenso en los mundos de arte (Becker, 2008). La participación de determinados artistas cuyas obras fueron valoradas especialmente y por tanto asociadas al talento de sus creadores, contribuía a colocar al disco de estos músicos locales en un lugar de mayor reconocimiento. Los artistas construían redes de colaboración que les permitían trabajos estables y constantes entre ellos. Devenir artista invitado en un disco generaba una posibilidad futura de volver a trabajar con dicho conjunto si la performance era exitosa.

Además de artistas invitados como cantantes, notamos que sólo en los dos últimos trabajos discográficos emergen voces femeninas. Esta situación no significa que las mujeres no hayan aparecido antes, pues pueden haber sido borradas en los relatos. Este artículo no profundiza sobre esta cuestión, pero llama la atención sobre el rol de las mujeres en los mundos de arte, ya sea porque ocuparon espacios estructuralmente diferenciados (McRobbie \& Garber, 2010), porque los mundos de arte diseñaban tecnologías del género que diferenciaba tareas de acuerdo al clivaje sexo-género (De Lauretis, 1989) y porque a partir de la instauración del patriarcado musical se ha construido históricamente una desigualdad de distribución de las habilidades artísticas (Green, 2001). 
En lo que respecta a los tópicos abordados por estas escasas canciones, notamos que tenían en común un alto grado de ambigüedad y vuelo poético. En aquellas composiciones no se hacía alusión a geografías específicas, pues no aparecían espacios concretos mencionados en las letras ni tampoco referencias temporales precisas. Esta característica se complementaba con las estéticas de las tapas donde tampoco es posible distinguir estas especificaciones, e incluso no siempre aparecen los cuerpos de los artistas. Esta cuestión contrasta con otra escena que analizamos para la tesis de doctorado, donde detectamos que un conjunto de artistas componía canciones que remitían a una estética que daba cuenta de espacios emblemáticos de la ciudad de Córdoba así como de hechos históricos que referían a la reciente experiencia dictatorial y las esperanzas que les generaba una nueva institucionalidad democrática en 1983 (Bruno, 2020). En cambio, las pocas canciones incluidas en los discos de Encuentro y Los Músicos del Centro, hallamos temáticas intimistas. Los narradores hablaban de rupturas amorosas y las sensaciones de vacío/ausencia que aquello les generaba, sobre la apatía y nuevos deseos de conexiones entre las personas, premoniciones oníricas, viajes de autoconocimiento, nuevas interpelaciones externas que generan nuevas sensaciones internas, descripciones climáticas de espacios naturales.

\section{Sobre sistemas de distribución y financiamiento}

Hasta aquí dimos cuenta de aspectos centrales de trayectorias de los artistas y obras que llegaron a ser plasmadas en discos de larga duración. En este apartado nos detenemos en los sistemas de distribución y financiamiento de esta escena de músicas instrumentales. De acuerdo a Howard Becker (2014) las formas de financiamiento de las obras de arte son un aspecto importante, que lleva a los propios músicos a clasificarse entre ellos de acuerdo al nivel de condescendencia a las demandas externas. En este sentido, las decisiones que tomaban los artistas para la financiación y distribución de sus obras repercutía en su propio prestigio y reputación. ¿Cómo era la relación de estos músicos instrumentales con las industrias culturales y los sellos grabadores? ¿Cómo diseñaban la distribución de sus obras para que sean apreciadas por sus públicos?

Un diacrítico importante que surgió en la tesis fue la distinción entre músicas comerciales y alternativas. De acuerdo a Simon Frith (2014) la caracterización de música comercial resulta de una práctica discursiva que valora la calidad estética de acuerdo al volumen de ventas. Que la música se venda en grandes cantidades resulta un éxito comercial y estético. Las músicas comerciales son financiadas por las industrias disqueras que posibilitan una gran cobertura de difusión, llegan a una gran porción de consumidores. Por otro lado, Frith plantea que las músicas comerciales tienen una intención de entretenimiento. Se presentan como una especie de escape de la vida rutinaria diaria de sus consumidores, al mismo tiempo que se integra a sus ritmos vitales, del trabajo y ocio.

De acuerdo al trabajo de campo que realizamos, los interlocutores coincidieron en delimitar una frontera que diferenciaba sus consumos y producciones: las músicas comerciales. Estas músicas eran una alteridad que definía el carácter de "alternativo" a las producciones que ellos creaban y consumían. Para los sujetos de nuestra investigación el mundo de la música comercial se integraba por artistas nacionales entre los cuales se destacaron los exponentes del Club del Clan y la posterior carrera solista de Palito Ortega. Las músicas comerciales sonaban en los boliches de moda y en la mayoría de las radios del país. Las músicas "alternativas" en cambio, tenían una difusión más modesta que generaba un grupo selecto de consumidores. Quienes eran cultores de estas sonoridades se imaginaban a sí mismos como una minoría, incluso capaces de reconocerse entre ellos. El acceso diferenciado y reservado para algunos entendidos les daba una característica especial. Encontrarse con sonoridades "alternativas" implicaba un esfuerzo y una búsqueda de parte de los interesados. En ocasiones los públicos tenían vínculos personales con los artistas, por tanto, las presentaciones en vivo o escucha de sus discos llegaba de la mano de amistades o romances. La música "alternativa" también tenía una ruta específica en determinados locales comerciales como peñas, bares y pubs.

A diferencia de las músicas comerciales, consumidores y productores de músicas "alternativas", diseñaron estrategias de distribución por fuera de las vías convencionales del mainstream. Cabe la aclaración que los artistas que indagamos en este artículo grabaron muchos de sus discos en sellos internacionales; esta situación no imposibilitaba tanto la adscripción a la categoría de alternativas a los grandes canales comerciales ni el diseño de sistemas de distribución autogestivos. Asimismo, advertimos que para la distribución de estas músicas instrumentales los discos no eran suficientes, ni tampoco el trabajo de promoción que los grandes sellos realizaban para estos artistas. También cabe mencionar que los discos grabados no eran el único formato posible para disfrutar de estas músicas, pues no todas las obras quedaban plasmadas en las grabaciones y solo podían apreciarse en vivo gracias a un circuito estable de locales mercantilizados que sostenían de manera periódica un espacio físico en la trama urbana de la ciudad.

La instancia de grabación de discos no era algo accesible a todos los músicos locales de una ciudad como Córdoba, dado que quienes financiaban estas producciones eran grandes industrias disqueras de capitales internacionales que tenían sus estudios y oficinas en la ciudad de Buenos Aires. Esta situación resultaba un obstáculo, pues requería de intermediarios que conocieran la producción local y recomendaran a los artistas a los empresarios que residían 
en la capital del país. Para el caso de los músicos que son objeto de este artículo, su pase hacia las compañías discográficas estuvo dada por la cercanía laboral de uno de los artistas con estas industrias. Julián Navarro trabajaba en la producción musical y conocía personal de sellos grabadores.

Los discos del grupo Encuentro y los Músicos del Centro se grabaron en la ciudad de Buenos Aires, los mismos artistas lograron un reconocimiento que les permitió desenvolverse como músicos sesionistas para otros proyectos también en la capital y en EEUU; sin embargo, no trasladaron su domicilio de la ciudad de Córdoba. Los interlocutores describieron a esta organización geográfica de los estudios y sellos discográficos como "centralismo porteño". Negarse a establecerse en la capital a pesar de la insistencia de sus productores fue para ellos una decisión con un alto grado de politicidad. Estos artistas querían romper con una tradición que señalaba que para tener éxito era necesario vivir en la capital del país, lo cual era calificado como un "sacrificio", una "traición" y un "desarraigo". Querían demostrar a integrantes de mundos musicales de Buenos Aires que en otros lugares del país también había producción musical con altos estándares estéticos que estaban a la altura de grabar sus propios discos.

Los artistas eran conscientes que los contratos con las compañías internacionales establecían una relación desigual. Los empresarios disqueros se quedaban con la mayor parte de la ganancia en el proceso de comercialización de las obras musicales. Si bien conocían el desequilibrio de las ganancias de las partes, eran escasas las posibilidades para evitar trabajar con estas grandes industrias. Por otro lado, si la grabación era un evento poco frecuente y muy caro en comparación con los ingresos de los artistas, y si tener un disco abría nuevas perspectivas de difusión de su trabajo que implicaba giras al interior provincial o incluso presentaciones en otras provincias; no resultaba extraño que los músicos aceptaran estos condicionamientos.

Como mencionamos en el apartado anterior, el grupo Encuentro y Los Músicos del Centro grabaron en sellos comerciales, sin embargo, hallamos una modificación a finales de la década de 1980 cuando los artistas iniciaron su trabajo con un sello independiente. A partir de 1989, Los Músicos del Centro editaron sus trabajos discográficos con el sello Melopea. Este sello tenía su sede en Buenos Aires e inició sus actividades en ese mismo año. Cabe considerar que en el año 1989 se registró una crisis híper inflacionaria que provocó una crisis política y social en el gobierno de Alfonsín que lo llevó a finalizar su gobierno de manera anticipada. En este contexto las industrias discográficas de capitales internacionales disminuyeron su producción para resguardar sus intereses económicos, la cancelación de contratos de los artistas menos reconocidos a nivel nacional, y por ello menos rentables fue parte de su estrategia de sobrevivencia. Esta coyuntura habilitó que otras formas de organización autogestiva cubrieran la demanda de conjuntos musicales locales que quedaron desprovistos de sellos para sus discos. ${ }^{15}$ Estos emprendimientos eran más modestos que los sellos internacionales, pues las obras que producían no tenían el mismo volumen de circulación y difusión.

En lo que respecta a la difusión y promoción, los artistas confeccionaban afiches y panfletos que distribuían en la vía pública. Esta publicidad de papel consistía en diseños sencillos, en general en blanco y negro, realizados a mano alzada o impresiones de bajo costo. Los artistas contaban con colaboradores, personas con un vínculo afectivo que prestaban su auto o los trasladaban a ellos y sus equipos para tocar o participaban con ellos en la tarea de pegar los afiches de difusión de sus presentaciones. Aquellas personas que actuaban como personal de apoyo, conocían a los artistas previamente, eran sus amigos, sus parejas o tenían relaciones de parentesco. Esta forma de trabajo generaba solidaridades mutuas y de apoyo entre los músicos. Muchas veces, a pesar que podían dedicarse a géneros musicales diferentes, se auxiliaban con equipos e instrumentos. También oficiaban de públicos, pues tenían vínculos afectivos con músicos de otros conjuntos que no eran los propios.

Esta forma de distribución de las músicas fomentaba una relación cercana entre artistas y sus públicos, donde ni los artistas eran "estrellas" ni los públicos "fanáticos" como si sucedía en los mundos de música comercial (Frith, 2014). Los artistas no se constituían como "estrellas inalcanzables" cuyos seguidores idolatraban y admiraban desde la distancia. Por el contrario, los músicos tenían una comunicación directa con las personas que asistían a ver sus shows, muchas veces entablaban conversaciones o intercambiaban información. Los artistas hablaban con sus públicos y quizás compartían alguna bebida antes y después de sus shows. Incluso, ciertos espacios facilitaban estos vínculos, pues no tenían escenarios elevados ni una salida diferenciada para los músicos. Las peñas y los pubs eran lugares pequeños, donde los shows tenían un intervalo que posibilitaba estas relaciones horizontales.

Otro aspecto que los diferenciaba de la música comercial, era la intención que los artistas decían tener respecto a sus obras y su distribución. Integrantes del Grupo Encuentro y de Los Músicos del centro no elegían su repertorio de acuerdo a potenciales ganancias monetarias y la cotización de sus músicas en el mercado del entretenimiento. Los artistas que son centro de este artículo, así como otros conjuntos que investigamos en la tesis construían una moralidad que daba forma

\footnotetext{
15 Otra estrategia que detectamos en la tesis fue que artistas de otra escena musical que analizamos tejieron alianzas con otra escena local de relevancia para concretar su trabajo discográfico. Se trataba de un conjunto de cuarteto con el cual los músicos compartían personal de apoyo y establecieron vínculos de confianza para concretar sus deseos.
} 
a las relaciones económicas que debían establecer con los bienes artísticos. ${ }^{16}$ Estos artistas profesaban como deseable la creación artística desligada de lo material, aunque efectivamente aquella división resultara artificial, pues en la distribución operaban las transacciones de dinero en la mayoría de los casos. Esta vinculación para con la economía de bienes culturales los diferenciaba de otros artistas y los colocaba en la posición de alternativos no comerciales. Sin embargo, esta, aparentemente, particular concepción sobre la motivación de sus acciones deslindada de los intereses materiales y económicos se extiende a otros casos etnográficos e históricos (Becker, 2014; Benzecry, 2012; Noel, 2012). Este precepto moral servía como criterio de identificación colectiva, y suponía que concebían deseable sujetarse a ciertos principios antes que a una racionalidad utilitarista que privilegiara la ganancia económica.

Si los músicos no asociaban la creación artística con la distribución masiva y ganancias materiales, establecían una jerarquía de valores en la cual la creación artística tomaba ciertas características particulares. Desde la perspectiva de sus creadores las obras sonoras que producían y consumían respondían a estándares de "calidad". Existía una serie de elementos a partir de los cuales se establecía esta jerarquía, si bien unos se vinculaban con la forma en que los artistas se relacionaban con los sistemas de financiamiento, otros se atribuían al producto estético en sí y la manera a través de la cual los artistas llegaban a aquellos resultados.

Los artistas instrumentales plantearon que la particularidad de las músicas que producían residía en altos estándares de calidad artística que tenían para sus oyentes y creadores. Esta situación las diferenciaba de las sonoridades de consumo masivo, pues estas últimas se caracterizaban por la simpleza técnica de sus armonías y arreglos. Las músicas que ellos producían, implicaban una destreza técnica y conocimiento de armonías complejas. Eran músicas consideradas "difíciles", que demandaban una gran preparación. Uno de los artistas explicó aquel estudio necesario para sus performances: "Lo que quiero decir es que, para tocar cosas, digamos, grosas de afuera, tenés que estar al nivel de poder hacerlo porque si no, no lo podés tocar" (Entrevista con M. Ingaramo). En este sentido, cabe recordar que estos productos artísticos eran mayormente instrumentales, lo cual subrayaba esta preparación técnica en la performance de instrumentos musicales, así como destrezas en arreglos, contrapuntos y ensambles de las obras. Si las letras y canciones no eran lo habitual, estos músicos privilegiaban el uso de sus instrumentos para evidenciar la calidad artística.

Si los artistas requerían una formación especial que les permitiera producir sonoridades que reconocían de "calidad" cabe preguntarnos sobre las formas de

16 Cabe señalar que el concepto de economía moral de Edward Thompson fue central para analizar estas prácticas (Thompson, 1995). preparación y estudio. Los artistas que son objeto de este artículo combinaron diferentes estrategias. Unos optaban por instituciones destinadas a la enseñanza de la música como el Conservatorio Superior de música Música Félix T. Garzón o la Escuela de Artes de la Universidad Nacional de Córdoba, carreras que no finalizaron. La mayoría de ellos estudiaban con profesores particulares, y luego devenían en autodidactas. Escuchaban mucha música y copiaban armonías "de oído". También aprendían con otros artistas que tenían mayor información, con los cuales compartían experiencias y proyectos en común. Interpretar y componer este tipo de músicas también implicaba rigurosidad en el trabajo de devenir músico y práctica diaria. Los artistas plantearon que para que sus grupos musicales tuvieran éxito eran necesario ensayos periódicos y reuniones a los fines de gestionar fechas, arreglos u otras cuestiones ligadas al trabajo de producción de sus conjuntos. Para que sus proyectos prosperaran resultaba menester actividades que ellos como artistas podían llevar a cabo y que refería a un ritmo de trabajo sostenido y disciplinado.

Los artistas de la escena instrumental que analizamos en este trabajo reconocían una carencia de inversión monetaria para el financiamiento de sus producciones. Ellos deseaban un incremento en los volúmenes monetarios de las músicas "alternativas", para lo cual anhelaban la existencia de empresarios que invirtieran en sus proyectos musicales. Los artistas soñaban con desentenderse de todo lo relativo a financiamiento y distribución, para dedicarse exclusivamente a la tarea creativa. Para estos músicos la carencia de recursos monetarios suficientes fue motivo para que sus proyectos no prosperaran y que orientaran sus actividades hacia otras opciones que le permitieran rentabilidad.

\section{Reflexiones finales}

En este artículo exploramos la trayectoria de dos conjuntos musicales que desarrollaron su obra artística entre los últimos años de la década de 1970 y 1990. Sin ánimos de dar por concluida la pesquisa, realizamos un ejercicio de delimitación posible que requerirá de nuevas indagaciones empíricas y analíticas. Acudimos a las categorías de Howard Becker a los fines de desglosar aspectos de una vasta red de cooperación que hacía posibles obras musicales en la ciudad de Córdoba en las décadas estudiadas. No obstante, los mundos del arte, como plantea el propio autor, no tienen fronteras fijas ni corresponde a quien las transforme en objeto de estudio establecer esos límites. Aunque en la tesis doctoral exploramos ciertas tramas de obras musicales, propusimos en este artículo una mirada situada a dos conjuntos que, por las características que describimos ubicamos en una posible escena instrumental. Esta escena instrumental constituye un objeto de pesquisa que será motivo de futuras investigaciones, aquí solo ensayamos unas primeras consideraciones. 
Como vimos, la existencia de esta escena no se circunscribe necesariamente a la ciudad de Córdoba o a los conjuntos que analizamos. La intención del artículo fue subrayar este dinamismo y algunas relaciones entre trayectorias particulares que deberán completarse con otros datos empíricos a construir. Hallamos ciertas pistas que nos llevaron a plantear como hipótesis que había una escena particular en la ciudad de Córdoba, ya sea por las características estéticas de las obras o por los procesos de producción que las hicieron posibles.

Los productos artísticos eran obras mayormente musicales sin letras cantadas. En caso de aparecer la palabra no se señalaban particularidades geográficas o temporales. Estos rasgos iban en sintonía con las gráficas de las tapas, que pocas veces mostraban a los autores de las obras. Los diseños también se realizaban en colaboración con artistas visuales del medio local e incluso internacional. Si bien estas descripciones requieren de mayor profundización analítica, pensamos que constituyen un diacrítico que diferenciaría estos artistas de otros de la ciudad.

Otra peculiaridad fue, que las tramas relacionales que configuraron los artistas indagados les permitieron una proyección internacional que resultó distintivo en comparación con otros artistas de la ciudad de Córdoba. Intentamos responder las razones que habilitaron esto, aunque no exploramos en detalle los tránsitos internacionales de los músicos. ¿Había otras obras/artistas que cumplían con ciertos estándares para devenir productos exportables? ¿Tenían características en común? ¿Quiénes y cómo se organizaban los agentes que financiaban estos productos internacionales?

En las trayectorias indagadas advertimos que había ciertas vinculaciones con espacios académicos, que sin embargo demandan mayor profundización. Por otro lado, resulta relevante a futuro considerar las variables históricas de un vasto periodo temporal que requiere de mayores sutilezas y análisis que aquí no fueron contempladas.

\section{Referencias Bibliográficas}

Acurso, M. B. (2016). Un mundo del folklore: Jóvenes y peñas en la ciudad de Córdoba. Trabajo Final de Maestría en Antropología. FFyh, UNC.

Alabarces, P. (1993). Entre gatos y violadores: El rock nacional en la cultura argentina (Vol. 3). Ediciones Colihue SRL.

Becker, H. (2008). Los mundos del arte. Universidad Nacional de Quilmes.

Becker, H., \& Faulkner, R. (2011). El jazz en acción. La dinámica de los músicos sobre el escenario. Buenos Aires: Editorial Siglo Veintiuno.
Becker, H. P. (2014). Outsiders hacia una sociología de la desviación. Siglo Veintiuno Editores.

Bennett, A., \& Peterson, R. A. (2004). Music scenes: Local, translocal and virtual. Vanderbilt University Press.

Benzecry, C. E. (2012). El Fanático de la Opera. Etnografía de una obsesión. Siglo XXI Editores.

Blázquez, G. (2008). Músicos, mujeres y algo para tomar: Los mundos de los cuartetos de Córdoba. Ediciones Recovecos.

Blázquez, G. (2014). ¡Bailaló!: Género, raza y erotismo en el cuarteto cordobés. Editorial Gorla.

Blázquez, G. (2019). Cooltura electrónica: Música, arte y subjetividades juveniles en Córdoba. Gorla.

Bruno, M. S. (2019). De "Aguas de la Cañada" a "Nada en la Cañada". Análisis de un mundo de canción urbana en la Córdoba de 1980 [Tesis de Doctorado en Ciencias Antropológicas]. Universidad Nacional de Córdoba.

Bruno, M. S. (2020). "Capítulo 11: Entre trovadores psicobolches y anarquistas. poéticas y políticas en un mundo musical de la Córdoba de 1980" en Celebrar. Una antropología de la fiesta y la performance. UNC.

Buch, E. (2016). Música, dictadura, resistencia: La Orquesta de París en Buenos Aires. Fondo de Cultura Económica.

Corti, B. (2015). Jazz argentino: La música" negra" del país" blanco". Gourmet Musical Ediciones.

Corti, B. (2018). "Sonar negro": La performance jazzística y la binarización de la "raza". Antecedentes en la ciudad de Buenos Aires.

De Lauretis, T. (1989). Tecnologías del género (Internet). Technologies of gender. Essays on theory, film and fiction. Londres....

Díaz, C. F. (2005). Libro de viajes y extravíos: Un recorrido por el rock argentino, 1965-1985. Narvaja.

Díaz, C. F. (2009). Variaciones sobre el" ser nacional": Una aproximación sociodiscursiva al" folklore" argentino. Ediciones Recovecos.

Elias, N. (1987). El proceso de la civilización: Investigaciones sociogenéticas y psicogenéticas. Fondo de Cultura Económica.

Elias, N. (1999). Mozart. Sociología de un genio. JSTOR. Frith, S. (2014). Ritos de la interpretación: Sobre el valor 
de la música popular. Grupo Planeta Spain.

Green, L. (2001). Música, género y educación. Morata.

Karush, M. (2017). Musicians in Transit. Argentina and the Globalization of Popular Music. Duke University Press.

Lenarduzzi, V. (2012). Placeres en movimiento: Cuerpo, música y baile en la" escena electrónica". El autor.

López, M. I. (2016). Abordajes de la improvisación en músicas ligadas al folklore en la escena de fusión argentina de los '80. XII Congreso IASPM-AL.

Lucero, P. (2009). Memorias de una ausencia. Armando Tejada Gómez y el Movimiento de la Nueva Canción [Tesis de Maestría en Comunicación y Cultura contemporánea]. Centro de Estudios Avanzados, UNC.

Manzano, V. (2011). Tiempos de contestación: Cultura del rock, masculinidad y política, 1966-1975. En Jóvenes en cuestión: Configuraciones de género y sexualidad en la cultura. Biblos.

McRobbie, Angela \& Garber, Angela. (2010). Chicas y subculturas: Una exploración. En Resistencia a través de rituales. Subculturas juveniles en la Gran Bretaña de la posguerra. Observatorio de Jóvenes, Comunicación y Medios. Facultad de Periodismo y Comunicación Social, Universidad Nacional de la Plata.

Molinero, C., \& Vila, P. (2014). A Brief Historyof the Militant Song in Argentina. En The Militant Song Movement in Latin America: Chile, Uruguay, and Argentina (pp. 193-228). Lexington Books.

Moyano, M. D. (2005). La producción plástica emergente en Córdoba (1970-2000). Ediciones del Boulevard.

Noel, G. (2012). Cuestiones disputadas. Repertorios morales y procesos de delimitación de una comunidad imaginada en la costa atlántica bonaerense. PUBLICAR-En Antropología y Ciencias Sociales, 11.

Pujol, S. (2011). Historia del baile: De la milonga a la disco.
Gourmet Musical.

Pujol, S. (2013). El jazz en la Argentina. Del paladar mundano al gusto local. En Jazz en español. Derivas hispanoamericanas (pp. 51-91). Universidad Veracruzana/ artefacto colectivo.

Pujol, S. A. (1992). Jazz al sur: La música negra en la Argentina. Emecé.

Pujol, S. A. (2005). Rock y dictadura: Crónica de una generación (1976-1983). Emecé.

Pujol, S. A. (2015). Oscar Alemán. La guitarra embrujada. Planeta.

Pujol, S. A. (2020). Quiero que la música hable de lo que pasa en Sudamérica. El giro tercermundista en el jazz de Leandro "Gato" Barbieri. Revista Argentina de Musicología, 21(1), 171-191.

Rodríguez, R. (2018). "Gracias por la musiquita". Una etnografía entre mujeres ['Trabajo Final de Licenciatura en Antropología]. Universidad Nacional de Córdoba.

Semán, P. (2012). Cumbia villera: Avatares y controversias de lo popular realmente existente. Nueva sociedad, 242, 149-161.

Semán, P., \& Vila, P. (1999). Rock chabón e identidad juvenil en la Argentina neoliberal. Los noventa. Política, sociedad y cultura en América Latina y Argentina de fin de siglo, 225-258.

Straw, Will. (1972). Communities and scenes in popular music. En The subcultures reader. Routledge.

Thompson, E. P. (1995). La economía "moral" de la multitud en la Inglaterra del siglo XVIII. En Costumbres en común. Crítica Barcelona.

Vittoreli, L. B. (2019). Rimas en el momento: Análisis etnográfico en la competencia de freestyle Sinescritura [Trabajo Final de Licenciatura en Comunicación Social]. Universidad Nacional de Córdoba. 